\title{
Genome-wide methylotyping resolves breast cancer epigenetic heterogeneity and suggests novel therapeutic perspectives
}

\author{
Alexander S Tanas¹,2, Vladimir O Sigin¹, Alexey I Kalinkin'1,3, Nikolai V Litviakov, Elena M \\ Slonimskaya ${ }^{5}$, Marina K Ibragimova ${ }^{4}$, Ekaterina O Ignatova ${ }^{6}$, Olga A Simonova ${ }^{1}$, Ekaterina \\ B Kuznetsova1,3, Tatiana V Kekeeva1, Sergey S Larin ${ }^{7,8}$, Elena V Poddubskaya9,10, Ivan D \\ Trotsenko ${ }^{11}$, Viktoria V Rudenko1', Kristina O Karandasheva ${ }^{1}$, Kseniya D Petrova1,12, Matvey \\ M Tsyganov ${ }^{4}$, Irina V Deryusheva ${ }^{4}$, Polina V Kazantseva ${ }^{5}$, Artem V Doroshenko ${ }^{5}$, Natalia A \\ Tarabanovskaya ${ }^{5}$, Galina G Chesnokova1, Marina I Sekacheva ${ }^{9}$, Marina V Nemtsova ${ }^{1,3}$, Vera \\ L Izhevskaya1, Sergey I Kutsev ${ }^{1,2}$, Dmitry V Zaletaev ${ }^{1,2,3}$ \& Vladimir V Strelnikov*,1,2 \\ ${ }^{1}$ Epigenetics Laboratory, Research Centre for Medical Genetics, Moscow, Russia \\ ${ }^{2}$ Molecular \& Cell Genetics Department, Pirogov Russian National Research Medical University, Moscow, Russia \\ ${ }^{3}$ Medical Genetics Laboratory, I.M. Sechenov First Moscow State Medical University (Sechenov University), Moscow, Russia \\ ${ }^{4}$ Laboratory of Oncovirology, Cancer Research Institute, Tomsk National Research Medical Center, Tomsk, Russia \\ ${ }^{5}$ Department of General Oncology, Cancer Research Institute, Tomsk National Research Medical Center, Tomsk, Russia \\ ${ }^{6}$ Clinical Pharmacology \& Chemotherapy, Federal State Budgetary institution «N.N. Blokhin National Medical Research Center of \\ Oncology» of the Ministry of Health of the Russian Federation, Moscow, Russia \\ ${ }^{7}$ Gene Therapy Laboratory, Institute of Gene Biology, Moscow, Russia \\ ${ }^{8}$ Molecular Immunology Laboratory, Federal Scientific Clinical Centre of Pediatric Hematology Oncology Immunology Named after \\ Dmitry Rogachev, Moscow, Russia \\ ${ }^{9}$ Clinic of Personalized Medicine, I.M. Sechenov First Moscow State Medical University (Sechenov University), Moscow, Russia \\ ${ }^{10}$ VitaMed LLC, Moscow, Russia \\ ${ }^{11}$ Institute of Medicine, RUDN University, Moscow, Russia \\ ${ }^{12}$ Department of Biological and Medical Physics, Moscow Institute of Physics \& Technology (State University), Dolgoprudny, \\ Moscow Region, Russia \\ *Author for correspondence: vstrel@list.ru
}

\begin{abstract}
Aim: To provide a breast cancer (BC) methylotype classification by genome-wide CpG islands bisulfite DNA sequencing. Materials \& methods: Xmal-reduced representation bisulfite sequencing DNA methylation sequencing method was used to profile DNA methylation of $110 \mathrm{BC}$ samples and 6 normal breast samples. Intrinsic DNA methylation BC subtypes were elicited by unsupervised hierarchical cluster analysis, and cluster-specific differentially methylated genes were identified. Results \& conclusion: Overall, six distinct $B C$ methylotypes were identified. BC cell lines constitute a separate group extremely highly methylated at the CpG islands. In turn, primary BC samples segregate into two major subtypes, highly and moderately methylated. Highly and moderately methylated superclusters, each incorporate three distinct epigenomic BC clusters with specific features, suggesting novel perspectives for personalized therapy.
\end{abstract}

First draft submitted: 5 December 2018; Accepted for publication: 22 January 2019; Published online: 7 February 2019

Keywords: 5-fluorouracil $\bullet$ adenylate cyclase pathway $\bullet$ breast cancer $\bullet$ cancer cell lines $\bullet$ DNA methylation $\bullet$ DNA methylotype $\bullet$ leukotriene receptors $\bullet$ reduced representation bisulfite sequencing $\bullet$ resveratrol $\bullet$ Xmal-RRBS

Breast cancer (BC) constitutes a clinically and biologically heterogeneous group of malignancies. Gene expression profiles specific for particular types of $\mathrm{BC}$ have been exhaustively studied, and at least four principal $\mathrm{BC}$ gene expression subtypes have been designated, luminal A, luminal B, HER2-enriched (HER2+) and basal-like or triple-negative $\mathrm{BC}$ (TNBC). BC classification based on gene expression profiling has improved on current standards for prognosis and prediction of chemotherapy benefit [1], yet relatively high cost of quantitative gene expression analysis and complicated mRNA processing may detain its translation into clinical practice, and more familiar clinically practical immunohistochemistry (IHC) assays are now widely used to distinguish BC subtypes [2]. Thus, although classification by gene expression is considered a gold standard in $\mathrm{BC}$ research, alternative approaches to 
classify BC samples based on the biological markers that might be handier in the clinical laboratories would be beneficial [3].

DNA methylation is among the most well-known factors of the gene expression regulation. Aberrant DNA methylation is associated with wide spectrum of disease and is most pronounced in malignancies. It was suggested that detection of the tumor-specific patterns of aberrant DNA methylation might be useful for early cancer diagnostics, for differential diagnostics of malignancies, and as predictive and prognostic markers [4-8].

Numerous studies of gene-specific epigenetic alterations associated with BC that have been undertaken, since the early 90 s of the past century, have revealed quite a number of genes abnormally methylated in BC. Abnormal methylation has been demonstrated for the genes involved in cell cycle regulation (CDKN2A, CDKN2B, p14/ARF, RB1, etc.), apoptosis (TP53, CDKN1A, HOX5, MDM2, DAPK1, TWIST1, TMS1 and FHIT), invasion and metastasis (CDH1, CDH13 and CTNB), receptor mediated signaling (ESR1, PR and RARB) and many others [915].

Since the middle of 2000s, researchers have focused on the associations of the methylation of the selected genes with $\mathrm{BC}$ subtypes $[16,17]$. At the same time, it became more and more obvious that the knowledge of cancer biology was still insufficient to confidently pick candidate regions, arguing for a more unbiased genomescale approach [18]. The earlier studies laid the foundation for the field and revealed the epigenetic alterations as hallmarks of cancer, as well as the crucial role of epigenetic mechanisms in tumorigenesis. Now, the introduction of next-generation sequencing (NGS) and array-based technologies for analyzing epigenetic states has accelerated our understanding about cancer [15]. Estimating recent advances in BC epigenomics, some experts postulate the 'epigenomic revolution in $\mathrm{BC}$ ', making a stress on the transition of the research from single-gene to genome-wide next-generation approaches [15]. Yet, while the array-based genome-wide DNA methylation studies of BC are already quite numerous, NGS-based studies are scarce (see Discussion for details). Taking into account some advantages of the sequencing data compared with the array datasets, we have developed a rapid and affordable genome-wide bisulfite DNA sequencing protocol, XmaI-reduced representation bisulfite sequencing (XmaI-RRBS) [19,20], and applied it to a significant collection of $\mathrm{BC}$ tissues and cells with the aim to provide a pilot $\mathrm{BC}$ methylotype classification based on the genome-wide $\mathrm{CPG}$ islands bisulfite sequencing data.

\section{Materials \& methods}

\section{Sample origin \& clinical characteristics}

A total of 105 BC samples, 6 autopsy samples of normal breast tissues and 5 BC cell lines (ZR751, HBL100, BT474, MCF7 и T47D) were analyzed. Biomaterial was provided by the Blokhin Russian Cancer Research Center, Gertsen Moscow Research Cancer Institute, Research Center for Medical Genetics, Institute of Gene Biology, Russian Scientific Center of Roentgenoradiology and Tomsk Cancer Research Institute.

\section{Cell lines}

According to Dai et al. 2017 [21], BC cell lines MCF7, T47D, ZR751 and BT474 present the following phenotypes: MCF7: ER+, PR+, HER2- (Luminal A)

T47D: ER+, PR+, HER2- (Luminal A)

ZR751: ER+, PR+/-, HER2- (Luminal A)

BT474: ER+, PR+, HER2+ (Luminal B)

HBL-100 is a spontaneously immortalized cell line derived from breast milk from a lactating, young, healthy woman [22]. However, these cells have SV40 sequences integrated within the genome and the DNA from these cells has transforming activity in vitro [23]. This cell line is molecularly classified as Basal B [24].

Thus, cell lines taken into our study belong to different molecular subtypes, luminal A, luminal B and supposedly, triple negative.

\section{Normal breast tissues}

Selection of normal controls for the cancer epigenomic research is challenging. Some authors postulate that healthy individuals who died of some traumatic event would have been the best sources for the tissues in such studies [25]. Yet, these types of samples are not readily available.

Byun et al. investigated the influence of the time periods between death and tissue collection (postmortem interval PMI: 44-1623 h), as well as the causes of death (particularly, cancer) on the integrity of tissue-specific and interindividual methylotypes. They have observed clustering of tissue-specific DNA methylation patterns, 
independent of PMI or the presence of cancer of another localization, suggesting that these two variables do not seriously distort genome-wide methylation patterns. Histologically, normal tissues from cancer patients were found to cluster with normal cancer-free tissues, independent of cancer status of the individual. An analysis of matched sets of lung cancers and adjacent normal lung tissue taken at the time of resection has demonstrated that normal cadaveric lung samples studied by Byun et al. cluster with the normal 'live' lung tissue data from patients with lung cancer [25].

In our study, we have selected six autopsy samples of normal breast tissues taken from the women without reported chronic disease, including cancers, who died of acute reasons. Morphological examination of the autopsy samples has ruled out the signs of BC. More importantly, this sampling of six autopsy normal breast tissues has previously been evaluated by us for the concordance of the genome-wide DNA methylation profiles with the normal breast samplings used in RRBS experiments within the ENCODE project [26], GEO Series GSE27584 and by Abba et al. [27], GEO Series GSE69994. Our study demonstrated high concordance, with Pearson correlation coefficient of 0.90 , for the methylotypes of the normal tissues under comparison [19].

\section{BC samples}

All tumors included in the study were not treated before sampling and were obtained either as biopsies prior to neoadjuvant treatment or as fragments of tumors removed surgically without prior neoadjuvant treatment. Informed consent was obtained from all patients and all work was carried out according to the permission from the Bioethics Committee at the Research Centre for Medical Genetics.

Tissue samples were frozen after collection and stored in liquid nitrogen until their transfer to the laboratory where they were constantly kept at $-80^{\circ} \mathrm{C}$. All tumor samples included in this study were collected between 2014 and 2018, using the same protocols established by the working group. As far as DNA methylation is a dynamic epigenetic mechanism, concerns may arise about the effects of technical factors on methylation, as this may confound results. Groen $e t$ al. have reviewed recent reports examining this issue in blood samples and concluded that variation in collection, storage and processing of blood DNA confers negligible effects on both global methylation and methylation status of specific genes. These results are concordant with studies that have investigated the effect of sample storage and processing on methylation in other tissues, such as tumor, sperm and placenta samples [28].

Relevant clinical and pathological information about BC patients and tumors that were included in this study is presented in Table 1. Taken that a number of samples were obtained before 2018, all tumors were classified according to the same 7 th edition of the AJCC cancer staging manual [29].

\section{DNA extraction \& Xmal-RRBS library preparation}

Genomic DNA was isolated from tissues and cell suspensions using a standard phenol:chloroform protocol after proteinase K treatment.

XmaI-RRBS library preparation was carried out exactly as described previously by Tanas et al. [19]. Briefly, genomic DNA digested with XmaI restriction endonuclease was partially filled-in with 5-methyl-dCTP by Klenow fragment $\left(3^{\prime}\right.$ to $5^{\prime}$ exo-). Partially filled-in DNA fragments were ligated with preannealed synthetic 5-methylcytosinecontaining adapters. Following ligation, unmethylated adaptor strands were nick translated by adding dATP, dTTP, dGTP and 5-methyl-dCTP, and Taq DNA polymerase. The resulting library fragments underwent size selection by the Pippin Prep (Sage Science) preparative electrophoresis according to the manufacturer's instructions to obtain a fraction of 181-311 bp fragments (186-316 bp in cases of barcoded adapters). Eluted fraction was bisulfite converted with the EpiTect Bisulfite Kit (Qiagen Cat\# 59104).

To prevent the $3^{\prime}$ ends of the degraded DNA fragments produced during bisulfite treatment from nonspecifically priming further polymerase reactions, we supplemented them with terminating nucleotides (ddNTPs) by use of SNaPshot Multiplex Kit (Thermo Fisher Scientific, MA, USA, Cat\# 4323163). Terminated DNA fragments were treated by RNAse A (Sigma-Aldrich, MO, USA, Cat\# 9001-99-4) and ALP (SibEnzyme Cat\# E328), in order to remove carrier RNA used in the EpiTect Bisulfite protocol and to dephosphorylate residual ddNTPs, respectively. An aliquot of the product was used to perform analytical qPCR in order to determine the upcoming preparative PCR threshold cycle. The final libraries were generated by PCR with the number of cycles defined for the sample via analytical qPCR (typically ranging from 12 to 18 cycles). The resulting libraries were purified and quantified on the Qubit 2.0 Fluorometer (Thermo Fisher Scientific), diluted to $100 \mathrm{pM}$, and libraries with unique barcodes were combined, if necessary. 
Table 1. The clinicopathological parameters of breast cancer patients, $n=105$.

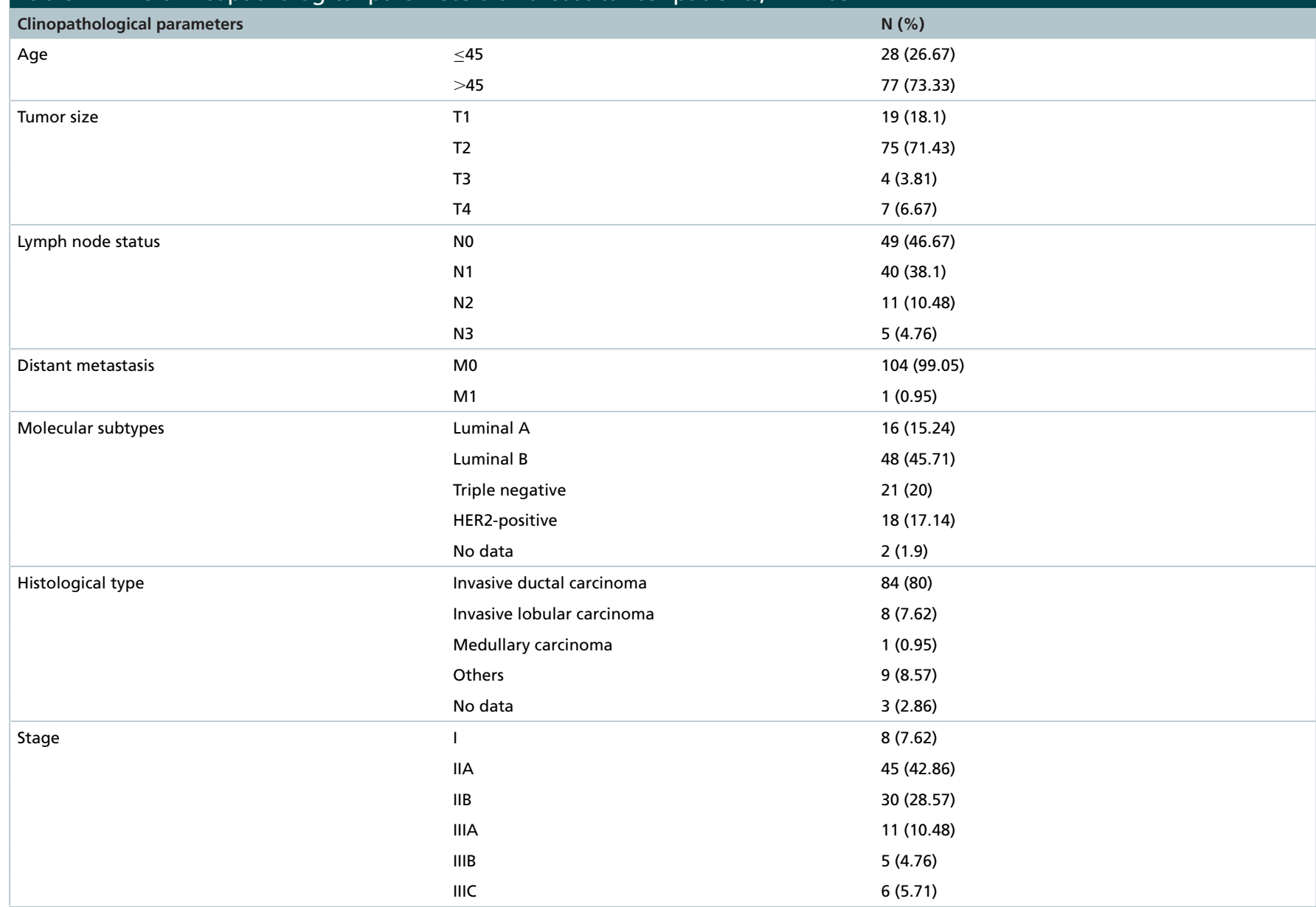

Emulsion PCR with the combined library was carried out with the Ion OneTouch instrument and Ion OneTouch 200 Template Kit (Thermo Fisher Scientific, Cat\# 4480974) according to the manufacturer's instructions. The resulting template-positive Ion Sphere Particles (ISPs) were enriched at $37^{\circ} \mathrm{C}$ using an Ion OneTouch ES system (Thermo Fisher Scientific).

\section{Sequencing \& alignment}

XmaI-RRBS libraries were sequenced on the Ion Personal Genome Machine (PGM) using the Ion PGM 200 Sequencing Kit and Ion 318 Chip (Thermo Fisher Scientific). It is of utmost importance to perform all procedures with ISPs from enrichment and till the chip installation into the PGM slot in a box preheated to $37^{\circ} \mathrm{C}$ in order to prevent mutual annealing of the ISPs. The sequence data processing, quality control and alignment of the XmaI-RRBS reads were performed exactly as described previously [19].

\section{Statistical analyses \& bioinformatics procedures}

Cluster analysis of the epigenome-wide data was performed using unsupervised hierarchical clustering. To perform the cluster analysis, we first selected 105,469 CpGs that in total possessed no more than 20\% missing data in the whole collection of 116 biological samples included in this study. Of these, we then selected high Shannon entropy CpGs using R package 'entropy' with 3.5 threshold. We have chosen Shannon entropy metrics in order to identify the top CpGs varying in the methylation status across the whole collection of samples under analysis, because it provides a more uniform distribution of $\mathrm{CpGs} \beta$-values variability in the sample collection than selection of differentially methylated CpGs by $\beta$-values standard deviation. The resulting 41,474 high entropy CpGs entered unsupervised hierarchical clustering with normalized pairwise Manhattan distance and ward. D2 agglomeration method [30]. No preliminary selection of a limited subset of the top CpGs found differentially methylated between 
Table 2. The list of genes that contain CpG dinucleotides discriminating the superclusters of highly and moderately methylated breast cancer samples.

\begin{tabular}{|c|c|}
\hline Related function of the encoded proteins & Genes \\
\hline Transcription regulation & $\begin{array}{l}\text { ACOT12, AEBP1, ATOH1, BARX1, BNC1, DLX6,EGR2, FOXC2, FOXE3, FOXO1B, GBX2, GFI1, GSX1, } \\
\text { HMGA2, HOXC5, HOXD3, HOXD4, IRX1, LHX3, MSC, NKX6-2, OLFM2, OLIG3, PAX7, PHOX2A, } \\
\text { POU4F1, PRDM14, PTF1A, RBFOX3, SALL3, SOX10, SOX18, SOX21, TBX18, TLX3, TWIST2, ZEB2, ZIC1, } \\
\text { ZIK1, ZNF512B, ZNF521 }\end{array}$ \\
\hline Metabolic enzymes and their regulators & $\begin{array}{l}\text { ADCY4, ADCY8, ADRA2A, ADORA2B,ADCYAP1, AKR1B1, ALPL, CDO1, CYP11A1, CYP26B1, DPEP1, } \\
D P Y S, E C E 2, G A D 2, G B G T 1, G L T 1 D 1, N G E F, S T 6 G A L N A C 5, T C E A 2, T T L L 8, \text { WBSCR17 }\end{array}$ \\
\hline Receptors and signal transduction & $\begin{array}{l}\text { AKAP2, EGFR, FNDC1, GLP1R, GNAT1, GPR158, GRIK1, IMPA1P, LTK, PRKCB, PTPRN, RALGDS, RGS7, } \\
\text { RSPO2, SFRP2 }\end{array}$ \\
\hline Transmembrane transporters and their regulators & $\begin{array}{l}\text { ANO4, ATP1A3, CNIH3, DPP6, KCNA3, KCNH8, KCNK10, KCNK17, KCNQ2, MFI2, NKAIN4, SLC38A4, } \\
\text { SLC9A3, SNAP25, RGS7 }\end{array}$ \\
\hline Immune response & CD8A, COLEC12, IRF4 \\
\hline Regulators of apoptosis & COMP, CARD14, NTN1, SHE, SHF, NGB \\
\hline Cell adhesion & ADAM19, CDH4,CHST11, EMILIN2, ITGA11, PDPN, SPON1, VWC2 \\
\hline Growth factors & FGF14, GDF10, NODAL, SCGB3A1 \\
\hline Chromatin remodeling & $A C T L 6 B$ \\
\hline Others & $\begin{array}{l}\text { ARL10, C1QL2, CELF2, COL9A3, DLEU7, DPYSL3, FSTL1, GABRG3, HAND2, HBM, KIAA1462, KIFC3, } \\
\text { ERICH1, ESYT3, HSPA12B, LHFPL3, LRRN4, MPPED1, MYOZ3, NRN1, PCBP3, PCSK5, PHACTR3, } \\
\text { PPP1R16B, RCSD1,SOBP,SPOCK2, PIF1, TERT, TDRD10, TMEM2OOB, TRABD, TRIM54, TSPAN11, UCN, } \\
\text { VSX1 }\end{array}$ \\
\hline Noncoding transcripts & $\begin{array}{l}\text { CLEC4GP1,DLX6-AS2, ERICH1-AS1, GPR158-AS1, HAND2-AS1, MIR10B, MIR1271, MIR615, MYO15B, } \\
\text { RN7SL121P, RNU1-143P, RPSAP52, SNAP25-AS1, SOX21-AS1, ZEB2-AS1 }\end{array}$ \\
\hline
\end{tabular}

breast tumors and normal breast samples has been made; normal samples were included in the complete dataset undergoing unsupervised hierarchical clustering. We then used the pvclust package in $\mathrm{R}$ to define statistically significant tumor clusters indicating patterns of potential biological relevance.

Differentially methylated CpGs discriminating BC epigenomic subtypes were selected with Mann-Whitney U-test. Differentially methylated genes (DMGs) were defined as those containing differentially methylated CpGs in a window of \pm 1000 base pairs around the transcription start site of the gene.

Enrichment of the DMGs sets with specific biological processes and molecular functions was assessed with the Gene Ontology (GO) Consortium Enrichment analysis tool [31,32]. GO features were considered significantly enriched at false discovery rate (FDR) $\mathrm{p}<0.05$ (Fisher's exact test).

Data processing scripts related to this project are available at http://www.epigenetic.ru/projects/rrbs-bc/

\section{Results}

Principal epigenomic subtypes (superclusters) of breast tissues \& cells defined by genome-wide CpG islands methylation patterns

We have obtained the XmaI-RRBS results for 105 BC samples, 5 BC cell lines and 6 normal breast samples (116 samples in total). The samples were processed as described in the 'Materials \& methods' section, and the average of $118 \mathrm{Mb}$ sequences with 500,000-2,000,000 reads per sample was obtained. Using the XmaI-RRBS ingenious workflow we achieved the highest currently possible efficiency of bisulfite-converted DNA sequences mapping against the reference genome, reaching $68 \%$.

Raw datasets have been submitted to The National Center for Biotechnology Information Gene Expression Omnibus (NCBI GEO) database, accession number GSE122799.

Unsupervised cluster analysis of the XmaI-RRBS results distinguishes two principal epigenomic subtypes of BC, highly and moderately methylated over the $\mathrm{CpG}$ islands, respectively (Figure $1 \&$ Table 2). Apart of these, BC cell lines constitute a unique separate cluster extremely highly methylated at the majority of the $\mathrm{CpG}$ islands. Although the studied cell lines were derived from the tumors belonging to different IHC types (luminal A, luminal B and triple-negative), their common property that clearly separates them from all surgically obtained $\mathrm{BC}$ specimens is an extremely hypermethylated (by CpG islands) phenotype.

We have identified the top 1000 high entropy $\mathrm{CpG}$ dinucleotides demonstrating most significant differences in the extent of their methylation between the highly and moderately methylated $\mathrm{BC}$ superclusters and found out that they belong to the promoter $\mathrm{CpG}$ islands of 163 genes (Table 2 \& Supplementary Figure 1). 


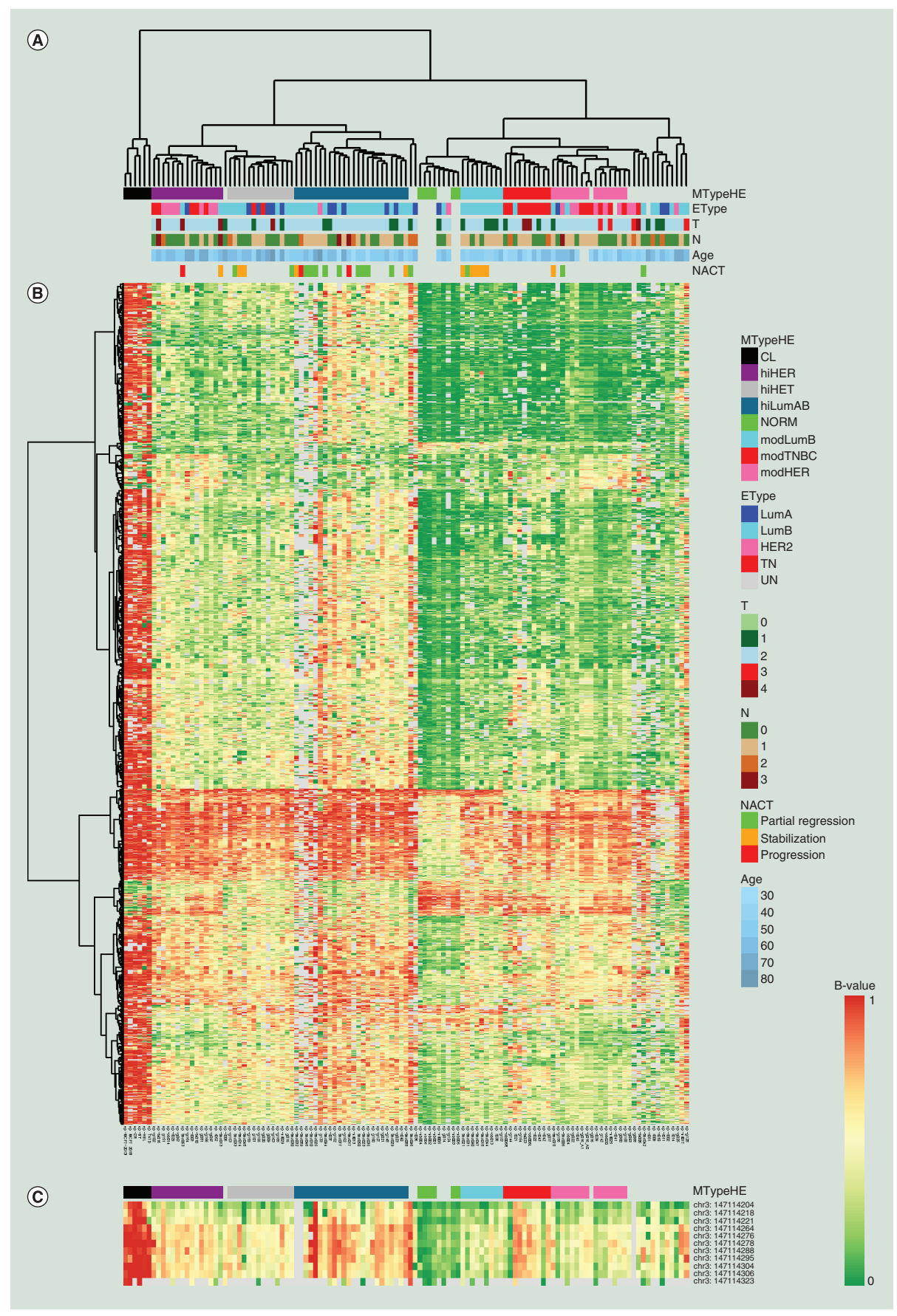

Figure 1. Cluster analysis of the Xmal-reduced representation bisulfite sequencing epigenome-wide data obtained for the breast cancer samples, breast cancer cell lines and normal breast samples. (A) The clusters underlined by the bars colored specifically for each of the eight breast MTypeHEs (methylotypes defined by the patterns of the high entropy CpGs), see the MTypeHE color legend (CL: cell line; NORM: normal breast tissue; modTNBC: moderately methylated triple-negative BC subtype; hiHET: highly methylated immunohistochemically heterogeneous subtype; modHER: moderately methylated HER2 + subtype; hiHER: highly methylated HER2+ subtype; hiLumAB: highly methylated luminal A\&B subtype; modLumB: moderately methylated luminal B subtype). BC samples' expression subtype (EType), patients' age, tumor size (T) and lymph node status $(\mathrm{N})$, response to the neoadjuvant chemotherapy (NACT) are also indicated as colored rectangles. Response to NACT is reported only for the cases where biopsies obtained prior to neoadjuvant treatment were used as a material for the methylotyping. All other BC samples in our study were the fragments of tumors removed surgically without prior neoadjuvant treatment. (B) The heat map for the specific sites in eight DNA methylation clusters. The red bars and green bars represent hypermethylation $\mathrm{CpG}$ sites and hypomethylation CpG sites, respectively. (C) The heat map for the CpG sites at the CpG71 island of the ZIC4 gene in eight DNA methylation clusters. A bar indicating the MTypeHE attribution of the samples is attached on the top of the heat map. Genomic (hg19/GRCh37) coordinates of the CpGs shown at the heat map are indicated at the right. Altogether, the methylation states of 11 sequential CpGs at the CpG71 island of the ZIC4 gene are shown. BC: Breast cancer. 
All the genes listed in Table 2 are hypermethylated in the highly methylated BC supercluster compared with the moderately methylated one. This gene list is enriched in such GO biological processes as positive regulation of transcription by RNA polymerase II, positive regulation of angiogenesis, organ morphogenesis, embryonic morphogenesis, tissue development and cell differentiation, in particular in neurogenesis. Enriched GO molecular functions include RNA polymerase II proximal promoter sequence-specific DNA binding and potassium channel activity.

In addition, manual data mining reveals that the highly/moderately methylated DMG's list is enriched in metabolic enzymes and their regulators. Among these, of note is frequent hypermethylation of the $\mathrm{CpG}$ islands of the genes coding for adenylate cyclases ADCY4, ADCY8 and other proteins involved in the adenylate cyclase-activating G-protein-coupled receptor signaling pathway (ADRA2A, ADORA2B, ADCYAP1, AKAP2), response to glucagon (GLP1R), glucose metabolism (AKR1B1). Hypermethylated in the supercluster of the highly methylated BCs is the ACOT12 gene encoding the acyl-CoA thioesterase 12, an enzyme that hydrolyzes acetyl-CoA to acetate and $\mathrm{CoA}$ (acetyl coenzyme A, a molecule that participates in many biochemical reactions in protein, carbohydrate and lipid metabolism). Judging by specific CpGs hypermethylation, one can hypothesize that the tumors constituting the hypermethylated supercluster might also be deficient in the regulation of the metabolic pathways related to pyruvate and several sulfurate compounds including sulfate, hypotaurine and taurine, as well as in the regulation of cellular cysteine concentrations (by possible inactivation of the cysteine dioxygenase gene CDOI). Of utmost biological and clinical significance might appear hypermethylation of the telomerase reverse transcriptase TERT gene, which we found specific for the hypermethylated BC supercluster.

\section{Breast tissues subtypes within the moderately methylated supercluster}

A moderately methylated BC supercluster falls apart into four clusters by the patterns of the promoter CpG islands methylation. Among these, normal breast tissues constitute the group of the least methylated samples. All normal breast samples cluster together with a tiny subset of BC samples that may either represent a genuine normal-like epigenomic subtype or, more likely, aggregate samples with extreme excess of normal cells, as far as these samples represent the tumors of different IHC subtypes. A normal/normal-like cluster is statistically significantly different from all the $\mathrm{BC}$ clusters by the absence of DNA methylation at the $\mathrm{CpG}$ islands of the genes shown on Supplementary Figure 2. Among the DMGs identified in our study, transcription factor ZIC4 appeared to be the most informative in distinguishing normal breast from $\mathrm{BC}$ samples, being abnormally methylated at its $\mathrm{CpG}$ island in $96 \%$ BC samples (with a more pronounced methylated patterns in the supercluster of highly methylated BCs), and not methylated in the normal breast tissues (Figure 1C).

Apart from a normal/normal-like cluster, the other three clusters that make up a moderately methylated supercluster are represented by the carcinoma samples belonging mainly to the three IHC subtypes, luminal B, TNBC and HER2+, with a more or less manifest grouping of the samples of a specific IHC subtype within each cluster (Figure 1).

A cluster of TNBC moderately methylated samples is discriminative from all other BC epigenomic subtypes by the nonmethylated CpG islands of 200 genes (Supplementary Figure 3), and by the methylated CpG islands of only a few, ADCY7, BPESC1, MECOM, PCBP3, PHACTR3, PRKCZ, PSPHP1, ZNF80 and ZNF831.

Among the genes unmethylated in this TNBC group in contrast with all other tumors are leukotriene receptor genes $L T B 4 R$ и $L T B 4 R 2$, as far as the roles of eicosanoids and, in particular, of leukotrienes in oncogenesis have recently attracted considerable interest. Leukotriene receptor inhibitors were proposed as potential anticancer drugs [33]. It has been demonstrated that antagonists of these receptors inhibit proliferation of $\mathrm{BC}$ cells in culture, and the effectiveness of antagonists correlates with leukotriene receptor expression [34]. In this respect, demethylated status of leukotriene receptors genes, if correlated with their elevated expression, might serve as useful markers of the tumor sensitivity to leukotriene receptors inhibitors [35]. In the light of our findings, TNBCs constituting a cluster of moderately methylated tumors might be more sensitive to leukotriene receptors inhibitors than the tumors belonging to other epigenomic subtypes in which the $\mathrm{CpG}$ islands of the corresponding genes are highly methylated. Interestingly, in this same TNBC group of tumors, the DPEP1 gene promoter is nonmethylated in contrast to all other $\mathrm{BC}$ epigenomic subtypes. The DPEP1 protein is known to regulate leukotriene activity by catalyzing the conversion of leukotriene D4 to leukotriene E4. The DMGs that might be related to the leukotriene receptors inhibitor cancer treatment and some other DMGs of potential significance for $\mathrm{BC}$ treatment identified in this study are exemplified in Table 3. 


\begin{tabular}{|c|c|c|c|}
\hline Gene(s) & $\begin{array}{l}\text { Promoter methylation in breast cancer } \\
\text { epigenomic subtypes }\end{array}$ & Function(s) of the encoded protein(s) & Therapeutic agent $^{\dagger}$ \\
\hline LTBAR and LTB4R2 & $\begin{array}{l}\text { Unmethylated in the moderately } \\
\text { methylated TNBC subtype }\end{array}$ & Leukotriene receptors & Leukotriene receptor inhibitors \\
\hline DPEP1 & $\begin{array}{l}\text { Unmethylated in the moderately } \\
\text { methylated TNBC subtype }\end{array}$ & $\begin{array}{l}\text { Regulates leukotriene activity by } \\
\text { catalyzing the conversion of } \\
\text { leukotriene D4 to leukotriene E4 }\end{array}$ & Leukotriene receptor inhibitors \\
\hline NME1 & $\begin{array}{l}\text { Unmethylated in the moderately } \\
\text { methylated TNBC subtype }\end{array}$ & $\begin{array}{l}\text { A pyrimidine diphosphate kinase } \\
\text { mediating the effects of } 5 \text {-fluorouracil } \\
\text { on DNA and RNA }\end{array}$ & 5-fluorouracil \\
\hline DPYS & $\begin{array}{l}\text { Hypermethylated in the highly } \\
\text { methylated luminal A\&B subtype }\end{array}$ & $\begin{array}{l}\text { Dihydropyrimidinase involved into the } \\
\text { 5-FU catabolism }\end{array}$ & 5-fluorouracil \\
\hline ERAS & $\begin{array}{l}\text { Hypermethylated in the moderately } \\
\text { methylated HER2 + subtype }\end{array}$ & $\begin{array}{l}\text { A constitutively active member of the } \\
\text { small GTPase Ras protein family }\end{array}$ & Ras inhibitors \\
\hline $\begin{array}{l}A D C Y 4, A D C Y 8 A D R A 2 A, A D O R A 2 B \\
A D C Y A P 1 \text { and } A K A P 2\end{array}$ & $\begin{array}{l}\text { Hypermethylated in the highly } \\
\text { methylated supercluster versus the } \\
\text { moderately methylated one }\end{array}$ & Adenylate cyclase pathway & Resveratrol \\
\hline
\end{tabular}

At least one more gene with the potential implication in pharmacogenetics, unmethylated in this TNBC cluster, encodes NME1, a pyrimidine diphosphate kinase mediating the effects of 5-fluorouracil on DNA and RNA [36,37]. Identification of differential methylation of this gene in our study requires more detailed research aimed to reveal its potential utility in predicting tumor sensitivity to 5-FU.

Another moderately methylated BC cluster includes the luminal B tumor samples exclusively. This cluster is specifically different from all other $\mathrm{BC}$ samples in a nonmethylated state of lots of genes (Supplementary Figure 4), and by relative hypermethylation of only a few, APOC1, BCORL1, C21orf2, C5orf66, CCDC85A, CST7, DNASE1L1, FBXL13, ISLR2, LARP4B, NFE4, NKAIN4, PITX1, SLC38A10, SLC4A11, TAZ, TMC6, TNFRSF25 and TSPYL6. Among the genes specifically nonmethylated in this group of moderately methylated luminal $\mathrm{B}$ tumors versus other BC samples, are at least two members of the superfamily of ATP-binding cassette transporters, $A B C A 3$ and $A B C A 7$. An ATP-binding cassette ransporter ABCA 3 constitutes resistance to xenobiotics, and active expression of its gene allowed by the nonmethylated status of the CpG island, might be associated with the tumor drug resistance [38].

The third cluster of moderately methylated BC samples is enriched in HER2+ tumors and, vice versa, all the moderately methylated HER2 + tumors fall exclusively into this cluster. The DMGs for this cluster are shown on Supplementary Figure 5. Interestingly, the moderately methylated HER2+ cluster is enriched with tumor samples in which the promoter $\mathrm{CpG}$ island of the ERAS gene is methylated. This feature discriminates moderately methylated HER2 + samples from all the other BCs, except the other HER2+ cluster, highly methylated, which is also enriched in the tumors with the methylated ERAS gene promoter. The ERAS gene encodes a constitutively active member of the small GTPase Ras protein family. The encoded protein activates the phosphatidylinositol 3-kinase signal transduction pathway in undifferentiated stem cells, but is not expressed in differentiated cells [39]. A recently published study reports ERAS expression in 10 out of $107 \mathrm{BC}$ samples. Six of these positive tumors were classified with a luminal A phenotype, two were luminal B and two were basal-like, which suggests that carcinomas with overexpression of ERAS mainly are luminal tumors [40]. This is consistent with our finding of the methylated status of the ERAS gene promoter CpG island in the HER2-positive samples, suggesting that this BC molecular subtype is not likely to express ERAS. It has already been suggested previously that ERAS oncogene expression might be regulated epigenetically, by histone acetylation [41].

Breast tissues subtypes within the highly methylated supercluster

The highly methylated supercluster unites mostly samples of the luminal and HER2+ tumors, with insignificant inclusion of TNBCs. This supercluster produces three clusters of the lower level, a HER2-positive-enriched, a luminal A\&B-enriched and a heterogeneous cluster of highly methylated BC samples. 
In contrast with the clusters of the moderately methylated samples, each cluster of the highly methylated samples is distinct from all the others in a hypermethylation of significant numbers of genes and a nonmethylated states of only few genes per cluster (Supplementary Figures 6-8).

The highly methylated luminal $A \& B$ cluster is specifically enriched in the hypermethylated CpG islands of the genes that may be associated with tumor invasion and metastasis, such as CDH4, CDHR5, ITGB4 and VIM. Yet, as far as our collection of samples is depleted in metastatic BCs (Table 1), we can only hypothesize the potential role of the hypermethylation of these genes in the progression of the highly methylated luminal A\&B tumors. This same cluster is notable for the abnormal methylation of the gene encoding a dihydropyrimidinase DPYS that is involved into the 5-FU catabolism [36], and its hypermethylation in one of the BC clusters identified in our study might be considered a marker of potential toxicity and chemotherapeutic response of breast tumors to 5-FU treatment.

\section{Discussion}

In this article, we present the first experience of obtaining RRBS data on a large collection of clinical samples by an XmaI-RRBS that was applied in order to effectively reduce the size of the NGS library without significant loss of the CpG islands. We have previously substantiated by in silico [20] and in vitro [19] experiments that sophisticated selection of the restriction endonuclease for use in the RRBS protocol allows to minimize the fraction of the uninformative repetitive sequences in the resulting pool of the RRBS library fragments. By using this approach, we have produced a considerable set of genome-wide bisulfite sequencing results related to normal and cancerous tissues and cells. This is the first experience of epigenomic subtyping of breast carcinomas based on the genome-wide bisulfite DNA sequencing. We have identified novel molecular subtypes of BC differing in the patterns of DNA methylation at the 1421 promoter $\mathrm{CpG}$ islands selected for evaluation from our XmaI-RRBS-derived dataset on the basis of containing high entropy $\mathrm{CpG}$ dinucleotides.

To our knowledge, to date seven studies identifying molecular genetic subtypes of $\mathrm{BC}$ based on the results of more or less genome wide DNA methylation assays have been published. The first was carried out with the use of the Illumina GoldenGate (Methylation Cancer Panel 1) on 28 BC samples. The authors have evaluated methylation of $\mathrm{CpG}$ dinucleotides belonging to 806 cancer related genes; for each of these genes methylation was studied for two $\mathrm{CpG}$ dinucleotides on average [3]. Three clusters of samples have been identified, corresponding to TNBC, HER2+ and luminal A tumors, respectively. Independently, another research group have analyzed 189 $\mathrm{BC}$ samples on the same Illumina GoldenGate platform [42]. They have also identified three BC clusters, although the clusters were enriched by luminal A, luminal B and TNBC types. HER2+ and normal-like samples appeared to be randomly distributed among the three epigenetic clusters.

Van der Auwera et al. have studied 19 inflammatory and 43 noninflammatory BC samples independently of immunohystochemical or gene expression subtypes by the Illumina Infinium Human Methylation27 BeadChip assay and have witnessed association of the inflammatory phenotype with the hypermethylated BC subtype [43].

In 2011, Kamalakaran et al. studied 108 BC samples with the hybridization array of their own design covering more than 27,000 CpG islands, and have designated three BC clusters, luminal $\mathrm{A} \& \mathrm{~B}$, normal-like and TNBCs clustering together with HER2+ tumors [44].

Ronneberg et al. published the results of the methylation analysis of 807 cancer related genes in $80 \mathrm{BC}$ samples. They have identified three clusters of samples, one of which united TNBCs and HER2+ tumors, while the two others were unique clusters both incorporating luminal A as well as luminal B samples [45].

The most representative in terms of the sample collection size study has been carried out under the network project for comprehensive BC molecular subtyping (The Cancer Genome Atlas Network, 2012). In this vast research, two platforms have been used to assess DNA methylation patterns, Illumina Infinium Human Methylation27 BeadChip (HM27) and Illumina Infinium Human Methylation450 BeadChip (HM450). HM450 is designed to analyze methylation of $482421 \mathrm{CpG}$ dinucleotides in $99 \%$ RefSeq genes (17 CpG dinucleotides per gene on average). Analysis of the results obtained for the same collection of samples with HM27 and HM450 has revealed significant discrepancies caused by batch effect as well as by technical interplatform differences. Considering this, the authors have selected for further clustering analysis the results obtained only from the most reliable probes. For this purpose, they have first selected only probes present in both assays $(\mathrm{N}=25,014)$. Next, the probes were removed for which experimental results could not be analyzed (approximately 20\%). Of the remaining, a half of the probes was discarded for the pronounced batch effect, and further, $90 \%$ were discarded for the inconsistency of the results demonstrated by the two assays. All the probes to the $\mathrm{CpG}$ dinucleotides mapped on the sex chromosome 
were discarded as well. Finally, the effective number of $\mathrm{CpG}$ dinucleotides that entered cluster analysis shrinked to $\mathrm{N}=574[46]$.

Cluster analysis of the methylation data obtained for thus selected $574 \mathrm{CpG}$ dinucleotides in a large collection of $466 \mathrm{BC}$ samples has revealed 5 groups of BC samples. One of the groups has incorporated near to all TNBC samples; the samples of other expression subtypes are exclusively rare in this cluster. The other four groups do not demonstrate any tendency to accumulate samples of a particular expression subtype. The number of HER2+ samples was insufficient to trace their tropism to one of the methylotypes, if any [46].

In 2014, Stefansson et al. published a genome wide methylation study performed on 40 BC samples and 17 normal breast tissue samples with the Infinium $450 \mathrm{~K}$ arrays. Similar to several earlier studies, three BC clusters have been designated, two clusters of luminal tumors and one cluster of TNBCs. Of the three HER2+ samples that entered the study, two settled in the TNBCs cluster [47].

The results of the microarray $\mathrm{BC}$ methylotyping studies described in this section may be summarized as follows. Normal and normal-like methylotypes are distinctly different from those of the luminal, HER2+ BCs and TNBCs. TNBCs demonstrate the most distinctive methylotype characterized by overall high similarity of the methylation patterns with the normal breast samples. Luminal type tumors segregate into more than one cluster, each of which may incorporate the samples of both luminal A and B subtypes. These clusters demonstrate pronounced differences between each other in the patterns of CpG methylation.

In this article, we present the first experience of genome-wide DNA methylation analysis of a significant collection of BC samples performed by a method alternative to the microarray hybridization, a high-throughput parallel bisulfite DNA sequencing. The clusters of BC samples calculated on the XmaI-RRBS data presented here generally recapitulate the conception of $\mathrm{BC}$ methylotypes established previously based on the array results.

For the first time in the same experimental series, we have assessed the methylomes of $\mathrm{BC}$ cell lines alongside with the surgically obtained $\mathrm{BC}$ samples and have demonstrated drastic difference of the former, independently of the IHC status of their origin, from the latter. BC cell lines genomes appeared to be pronouncedly hypermethylated at the majority of the $\mathrm{CpG}$ islands compared with those of the primary tumors. Earlier, similar results were obtained in a study of $\mathrm{CpG}$ island promoter hypermethylation of 15 tumor suppressor genes in human cancer cell lines [48]. Our findings clearly demonstrate that the cell line methylotypes are not representative of human live tissues, or of any cancer type, and should be used at least extremely cautiously in cancer research. Yet, as far as the human cancer cell lines are widely used and standardized objects, we suggest that their deep methylotyping will provide a basis for their more sophisticated use in basic and translational research with respect to their DNA methylation environment.

\section{Conclusion}

The study presented here provides a pilot version of the $\mathrm{BC}$ epigenomic classification based on the genome-wide bisulfite DNA sequencing. Although the necessity of its validation by independent research groups is of importance, we suggest that it already reveals the basics of BC heterogeneity at the epigenomic (methylotypic) level and provides a valuable source of DMGs that may be further thoroughly evaluated in terms of their relation to cancer biology and treatment.

\section{Future perspective}

With the introduction of genome-wide analysis techniques, basic cancer genetics has finally progressed from 'searching under the lantern' to truly unbiased, hypotheses-free research methods that possess a rich potential to unravel a wide layer of knowledge hitherto otherwise inaccessible. Cancer epigenomics, including DNA methylomics, has somewhat lagged behind the structural genomics and transcriptomics, probably due to technical complexity of genome-wide bisulfite DNA sequencing destined to be the gold standard in DNA methylomics. In the near future, with more and more affordable and technically sound protocols of genome-wide bisulfite DNA sequencing and analysis, we will face rapid accumulation of novel valuable data on tumor biology derived from the epigenomic studies, that will facilitate the development of previously unexpected approaches to cancer diagnostics and treatment. 
Financial \& competing interests disclosure

This research was supported by the Russian Science Foundation (project No.18-15-00430). The authors have no other relevant affiliations or financial involvement with any organization or entity with a financial interest in or financial conflict with the subject matter or materials discussed in the manuscript apart from those disclosed.

No writing assistance was utilized in the production of this manuscript.

\section{Ethical disclosure of conduct}

For studies involving data relating to human or animal experimental investigations, appropriate institutional review board approval has been obtained and is described within the article (for those investigators who do not have formal ethics review committees, the principles outlined in the Declaration of Helsinki have been followed)

\section{Open access}

This work is licensed under the Attribution-NonCommercial-NoDerivatives 4.0 Unported License. To view a copy of this license, visit http://creativecommons.org/licenses/by-nc-nd/4.0/

\section{Summary points}

- Breast cancer (BC) classification based on gene expression profiling has improved on current standards for prognosis and prediction of chemotherapy benefit, yet relatively high cost of quantitative gene expression analysis and complicated mRNA processing may detain its translation into clinical practice.

- Although classification by gene expression is considered a gold standard in BC research, alternative approaches to classify BC samples based on the biological markers that might be handier in the clinical laboratories would be beneficial. Differential DNA methylation widely represented in cancers promises to provide such markers for clinically useful BC classification.

- Development of epigenetic BC classification is accelerated by the introduction of the next-generation sequencing and array-based technologies for analyzing epigenetic states; yet, while the array-based genome-wide DNA methylation studies of BC are already quite numerous, NGS-based studies are scarce.

- We present a pilot BC methylotype classification based on the genome-wide CpG islands bisulfite sequencing data obtained with the Xmal-reduced representation bisulfite sequencing on a collection exceeding a hundred BC samples.

- Unsupervised cluster analysis of the Xmal-reduced representation bisulfite sequencing results distinguishes two principal epigenomic subtypes (superclusters) of BC, highly and moderately methylated over the promoter CpG islands, respectively.

- $\mathrm{BC}$ cell lines constitute a unique separate cluster extremely highly methylated at the majority of the promoter CpG islands.

- A moderately methylated BC supercluster falls apart into four clusters by the patterns of the promoter CpG islands methylation, with the normal breast tissues constituting the group of the least methylated samples.

- The three moderately methylated $B C$ clusters are represented by the carcinoma samples belonging mainly to the three immunohistochemistry (IHC) subtypes, luminal B, triple-negative BC and HER2+, with a more or less manifest grouping of the samples of a specific IHC subtype within each cluster.

- The highly methylated supercluster unites mostly samples of the luminal and HER2+ tumors, with insignificant inclusion of triple-negative BCs; this supercluster produces three clusters of the lower level, a HER2-positive-enriched, a luminal(A\&B)-enriched, and an IHC heterogeneous cluster of highly methylated BC samples.

- Differentially methylated genes identified in this study might suggest novel therapeutic perspectives for BC. Some examples are the NME1 and DPYS genes mediating the effects of 5-fluorouracil; LTB4R, LTB4R2 and DPEP1 genes that might modulate the effects of the leukotriene receptor inhibitors; elements of the adenylate cyclase pathway that may constitute sensitivity to resveratrol.

\section{References}

Papers of special note have been highlighted as: $\bullet$ of interest; $\bullet \bullet$ of considerable interest

1. Parker JS, Mullins M, Cheang MC et al. Supervised risk predictor of breast cancer based on intrinsic subtypes. J. Clin. Oncol. 27(8), 1160-1167 (2009).

-• Introduces a presently widely used 50-gene breast cancer subtype predictor with the goal to improve on the standards for breast cancer prognosis and prediction of chemotherapy benefit by developing a risk model that incorporates the gene expression-based 'intrinsic' subtypes luminal A, luminal B, HER2-enriched and basal-like. 
2. Cheang MC, Chia SK, Voduc D et al. Ki67 index, HER2 status, and prognosis of patients with luminal B breast cancer. J. Natl Cancer Inst. 101(10), 736-750 (2009).

3. Bediaga NG, Acha-Sagredo A, Guerra I et al. DNA methylation epigenotypes in breast cancer molecular subtypes. Breast Cancer Res. 12(5), R77 (2010).

4. Zemlyakova VV, Zhevlova AI, Zborovskaya IB et al. Methylation profile of several tumor suppressor genes in non-small-cell lung cancer. Mol. Biol. 37, 836-840 (2003).

5. Zemlyakova VV, Strel'nikov VV, Zborovskaya IB et al. Comparison of aberrant methylation of CpG Islands in the p16/CDKN2A and p14/ARF promoters in non-small cell lung cancer and acute lymphoblastic leukemia. Mol. Biol. 38, 821-827 (2004).

6. Zaletaev DV, Nemtsova MV, Strelnikov VV et al. Diagnostics of epigenetic alterations in hereditary and oncological disorders. Mol. Biol. 38, 174-182 (2004).

7. Esteller M. Cancer epigenomics: DNA methylomes and histone-modification maps. Nat. Rev. Genet. 8(4), 286-298 (2007).

- A concise yet comprehensive review conciliating classical postulates of cancer epigenetics with the perspective of epigenomic research.

8. Golovastova MO, Tsoy LV, Bocharnikova AV et al. The cancer-retina antigen recoverin as a potential biomarker for renal tumors. Tumor Biol. 37, 9899-9907 (2016).

9. Zemlyakova VV, Zhevlova AI, Strelnikov VV et al. Abnormal methylation of several tumor suppressor genes in sporadic breast cancer. Mol. Biol. 37, 591-597 (2003).

10. Kuznetsova EB, Kekeeva TV, Larin SS et al. Methylation of the BIN1 gene promoter CpG island associated with breast and prostate cancer. J. Carcinog. 6(1), 9 (2007).

11. Kuznetsova EB, Kekeeva TV, Larin SS et al. Novel markers of gene methylation and expression in breast cancer. Mol. Biol. 41, 562-570 (2007).

12. Simonova OA, Kuznetsova EB, Poddubskaya EV et al. DNA methylation in the promoter regions of the laminin family genes in normal and breast carcinoma tissues. Mol. Biol. 49, 598-607 (2015).

13. Tanas AS, Shkarupo VV, Kuznetsova EB et al. Novel tools for unbiased DNA differential methylation screening. Epigenomics 2(2), 325-333 (2010).

14. Strelnikov V, Tanas A, Shkarupo V et al. Non-microarray DNA differential methylation screening in breast cancer. Cancer Genet. Cytogenet. 203(1), 93 (2010).

15. Davalos V, Martinez-Cardus A, Esteller M. The epigenomic revolution in breast cancer: from single-gene to genome-wide next-generation approaches. Am. J. Pathol. 187(10), 2163-2174 (2017).

16. Fiegl H, Millinger S, Goebel G et al. Breast cancer DNA methylation profiles in cancer cells and tumor stroma: association with HER-2/neu status in primary breast cancer. Cancer Res. 66(1), 29-33 (2006).

17. Feng W, Shen L, Wen $\mathrm{S}$ et al. Correlation between CpG methylation profiles and hormone receptor status in breast cancers. Breast Cancer Res. 9(4), R57 (2007).

18. Bock C. Epigenetic biomarker development. Epigenomics 1(1), 99-110 (2009).

-• Outlines a systematic approach to epigenetic biomarker development and highlights key bioinformatic tools that facilitate discovery, optimization and validation of novel biomarkers.

19. Tanas AS, Borisova ME, Kuznetsova EB et al. Rapid and affordable genome-wide bisulfite DNA sequencing by XmaI-reduced representation bisulfite sequencing. Epigenomics 9(6), 833-847 (2017).

- Provides a comprehensive description and substantiation of the XmaI-reduced representation bisulfite sequencing approach.

20. Tanas AS, Kuznetsova EB, Borisova ME et al. Reduced representation bisulfite sequencing design for assessing the methylation of human CpG islands in large samples. Mol. Biol. 49, 618-626 (2015).

21. Dai X, Cheng H, Bai Z, Li J. Breast cancer cell line classification and its relevance with breast tumor subtyping. J. Cancer 8(16), 3131-3141 (2017).

22. Gaffney EV. A cell line (HBL-100) established from human breast milk. Cell Tissue Res. 227(3), 563-568 (1982).

23. Vanhamme L, Szpirer C. Transforming activity of the human mammary line HBL100 DNA is associated with SV40 large T antigen genetic information integrated in its genome. Carcinogenesis 9(4), 653-655 (1988).

24. Chavez KJ, Garimella SV, Lipkowitz S. Triple-negative breast cancer cell lines: one tool in the search for better treatment of triple-negative breast cancer. Breast Dis. 32(1-2), 35-48 (2010).

25. Byun HM, Siegmund KD, Pan F et al. Epigenetic profiling of somatic tissues from human autopsy specimens identifies tissue-and individual-specific DNA methylation patterns. Human Mol. Genet. 18(24), 4808-4817 (2009).

26. Wang H, Maurano MT, Qu H et al. Widespread plasticity in CTCF occupancy linked to DNA methylation. Genome Res. 22, 1680-1688 (2012).

27. Abba MC, Gong T, Lu Y et al. A molecular portrait of high-grade ductal carcinoma in situ. Cancer Res. 75, 3980-3990 (2015). 
28. Groen K, Lea RA, Maltby VE, Scott RJ, Lechner-Scott J. Letter to the editor: blood processing and sample storage have negligible effects on methylation. Clin. Epigenet. 10(1), 22 (2018).

29. Edge SB, Compton CC. The American Joint Committee on Cancer: the 7 th edition of the AJCC cancer staging manual and the future of TNM. Ann. Surg. Oncol. 17(6), 1471-1474 (2010).

30. Clifford H, Wessely F, Pendurthi S, Emes RD. Comparison of clustering methods for investigation of genome-wide methylation array data. Front. Genet. 2, 88 (2011).

31. Ashburner M, Ball CA, Blake JA et al. Gene ontology: tool for the unification of biology. Nat. Genet. 25(1), 25-29 (2000).

32. Gene Ontology Consortium. Expansion of the gene ontology knowledgebase and resources. Nucleic Acids Res. 45(D1), D331-D338 (2016).

33. Wang D, DuBois RN. Eicosanoids and cancer. Nat. Rev. Cancer 10(3), 181-193 (2010).

- Highlights the intricate roles of eicosanoids in epithelial-derived tumors and their microenvironment. Understanding the molecular mechanisms underlying the role of eicosanoids in cancer progression will help to develop more effective cancer chemopreventive and/or therapeutic agents.

34. Park GS, Kim JH. Myeloid differentiation primary response gene 88-leukotriene B4 receptor 2 cascade mediates lipopolysaccharide-potentiated invasiveness of breast cancer cells. Oncotarget 6(8), 5749-5759 (2015).

35. Kalinkin AI, Strelnikov VV, Ignatova EO et al. Leukotriene B4 receptors as a therapeutic target for triple-negative breast cancer. Ann. Oncol. 29(Suppl. 6), mdy314-mdy019 (2018).

36. Muhale FA, Wetmore BA, Thomas RS et al. Systems pharmacology assessment of the 5-fluorouracil pathway. Pharmacogenomics 12(3), 341-350 (2011).

37. Strelnikov VV, Ignatova EO, Kekeeva TV et al. Epigenomics paves a new way to assess potential toxicity and chemotherapeutic response of breast tumours to 5-fluorouracil. Ann. Oncol. 29(Suppl. 6), mdy318-mdy016 (2018).

38. Litviakov NV, Cherdyntseva NV, Tsyganov MM et al. Deletions of multidrug resistance gene loci in breast cancer leads to the down-regulation of its expression and predict tumor response to neoadjuvant chemotherapy. Oncotarget 7(7), 7829-7841 (2016).

39. Liu Y, Wang Z, Li H et al. Role of the ERas gene in gastric cancer cells. Oncol. Rep. 30(1), 50-56 (2013).

- This article not only provides experimental evidence of the ERAS gene expression role in the cancer cells proliferation and metastasis, but also presents a concise review of the history of ERAS discovery and research.

40. Suárez-Cabrera C, de la Peña B, González LL et al. The Ras-related gene ERAS is involved in human and murine breast cancer. Sci. Rep. 8(1), 13038 (2018).

41. Yasuda K, Yashiro M, Sawada T, Ohira M, Hirakawa K. ERas oncogene expression and epigenetic regulation by histone acetylation in human cancer cells. Anticancer Res. 27(6B), 4071-4075 (2007).

42. Holm K, Hegardt C, Staaf J et al. Molecular subtypes of breast cancer are associated with characteristic DNA methylation patterns. Breast Cancer Res. 12(3), R36 (2010).

43. Van der Auwera I, Yu W, Suo L et al. Array-based DNA methylation profiling for breast cancer subtype discrimination. PLoS ONE 5(9), e12616 (2010).

44. Kamalakaran S, Varadan V, Giercksky Russnes HE et al. DNA methylation patterns in luminal breast cancers differ from non-luminal subtypes and can identify relapse risk independent of other clinical variables. Mol. Oncol. 5(1), 77-92 (2011).

45. Rønneberg JA, Fleischer T, Solvang HK et al. Methylation profiling with a panel of cancer related genes: association with estrogen receptor, TP53 mutation status and expression subtypes in sporadic breast cancer. Mol. Oncol. 5(1), 61-76 (2011).

46. Cancer Genome Atlas Network. Comprehensive molecular portraits of human breast tumours. Nature 490(7418), 61-70 (2012).

47. Stefansson OA, Moran S, Gomez A et al. A DNA methylation-based definition of biologically distinct breast cancer subtypes. Mol. Oncol. 9(3), 555-568 (2015).

48. Paz MF, Fraga MF, Avila S et al. A systematic profile of DNA methylation in human cancer cell lines. Cancer Res. 63(5), 1114-1121 (2003). 
